\title{
FINITE UNIONS OF OVERRINGS OF AN INTEGRAL DOMAIN
}

\author{
Christian Gottlieb \\ Department of Mathematics, University of Stockholm \\ SE-106 91 Stockholm, Sweden \\ gottlieb@math.su.se
}

\begin{abstract}
Let $R$ be an integral domain, and let $A, A_{1}, A_{2}, \ldots, A_{S}$ be overrings of $R$, where $A$ is of the form $S^{-1} R$, where $S=R \backslash \mathfrak{p}_{1} \cup \cdots \cup \mathfrak{p}_{n}$ for some prime ideals $\mathfrak{p}_{i}$, and where each $A_{i}, i \geq 2$, is of the form $S_{i}^{-1} R$ for some multiplicatively closed subset $S_{i}$ of $R$. It is shown that if $A \subseteq A_{1} \cup \cdots \cup A_{s}$, then $A \subseteq A_{i}$ for some $i$.
\end{abstract}

This investigation was initiated and inspired by a theorem of A. Azarang, proved as Corollary 3.10 in [1]. The theorem, by Azarang properly called the valuation avoidance lemma, states that if $V, V_{1}, V_{2}, \ldots, V_{s}$ are valuation rings with a common field of fractions $K$, and if $V \subseteq V_{1} \cup \cdots \cup V_{s}$, then in fact $V \subseteq V_{i}$ for some $i$. In other words, if $V$ avoids every $V_{i}$ (i.e. $V \nsubseteq V_{i}$ for every $i$ ), then $V$ avoids the union of the $V_{i}$ :s (i.e., $V \nsubseteq V_{1} \cup V_{2} \cup \cdots \cup V_{s}$ ).

We have previously studied avoidances of ideals and modules over commutative rings in a few papers, the most recent being [2], where a list of further references is given. One of the earliest papers, and an excellent source of inspiration, on finite unions of ideals and modules is the paper by N. McCoy [5].

By developing the techniques used in [2] a bit further, we shall be able to generalize Azarang's result to the following situation. $R$ is an integral domain. $A$ and $A_{1}, A_{2}, \ldots$, 
$A_{s}$ are overrings of $R$, i.e. rings between $R$ and the field of fractions $K$ of $R$. For $s \geq 2$ we suppose that $A_{i}$ is a ring of fractions of $R$, say $A_{i}=S_{i}^{-1} R$. Also we suppose that $A=$ $R_{\mathfrak{p}_{1} \cup \ldots \cup \mathfrak{p}_{n}}$ where the $\mathfrak{p}_{i}$ are prime ideals, i.e., that $A=S^{-1} R$ where $S$ is the complement of $\mathfrak{p}_{1} \cup \cdots \cup \mathfrak{p}_{n}$. Then if $A \subseteq A_{1} \cup A_{2} \cup \cdots \cup A_{s}$, in fact $A \subseteq A_{i}$ for some $i$. This is Theorem 6 below.

It is an advantage to formulate some of our results for modules and then apply these results to rings, so we begin by turning our attention to modules. In the sequel $M, N$ and so on will always denote modules over a commutative ring $R$.

Speaking informally one might say that there are two kinds of avoidances of modules, each with corresponding avoidance-lemmas, namely the following:

(i) the fact that a single module $M$ avoids each module in a set $M_{1}, M_{2}, \ldots, M_{n}$ of modules, does under certain conditions imply that $M$ avoids the union $M_{1} \cup \cdots \cup M_{n}$ of the modules, i.e., $M \nsubseteq M_{1} \cup \cdots \cup M_{n}$

(ii) the fact that each module in a set $M_{1}, M_{2}, \ldots, M_{n}$ of modules avoids a single module $M$, does under certain conditions imply that the intersection $M_{1} \cap \cdots \cap M_{n}$ avoids $M$, i.e., $M_{1} \cap \cdots \cap M_{n} \nsubseteq M$.

An example of a theorem of the first kind, is the usual prime avoidance lemma: If an ideal $\mathfrak{a} \subseteq \mathfrak{p}_{1} \cup \cdots \cup \mathfrak{p}_{n}$, where the $\mathfrak{p}_{i}$ are prime, then $\mathfrak{a} \subseteq \mathfrak{p}_{i}$ for some $i$. Another example is of course Azarang's result. Note also that for $n=2$ we have avoidance of the first kind without any assumptions on $M_{1}$ and $M_{2}$.

An example of a theorem of the second kind, is the still more elementary fact, that if the intersection of ideals $\mathfrak{a}_{1} \cap \cdots \cap \mathfrak{a}_{n} \subseteq \mathfrak{p}$, where $\mathfrak{p}$ is prime, then some $\mathfrak{a}_{i} \subseteq \mathfrak{p}$.

The two kinds of avoidances are related, as can be seen from the usual proof of the prime avoidance lemma. This is also illustrated by the following lemma, which is [2, Corollary 4], and which will be used in the proof of Proposition 3 below.

Lemma 1. Suppose $s \geq 2$ and $N \subseteq N_{1} \cup \cdots \cup N_{s}$ but $N \nsubseteq N_{2} \cup \cdots \cup N_{s}$. Then $N \cap N_{2} \cap N_{3} \cap \cdots \cap N_{s} \subseteq N_{1}$.

In [2], following the terminology of Heinzer-Ratliff-Rush in [3], a module $N$ over a commutative ring was said to be strongly irreducible if $N \supseteq N_{1} \cap N_{2}$ implies that $N \supseteq N_{1}$ or $N \supseteq N_{2}$. By induction this extends to finite intersections. Note that this property relates to avoidance of the second kind. The most obvious example of strongly irreducible modules are prime ideals. Modules, which are finite intersections of strongly irreducible modules, were in [2] said to be pseudo-radical, in analogy with radical ideals, which by definition are intersections of prime ideals.

We will extend the notions of strongly irreducible and pseudo-radical, and prove some general results. These will then be applied in the special case, where $R$ is an integral domain, and the modules are overrings of $R$.

Our main result in [2] was that if $N \subseteq N_{1} \cup \cdots \cup N_{s}$, where all $N_{i}$ except possibly two are pseudo-radical, then $N \subseteq N_{i}$ for some $i$. Informally speaking: from a condition related to avoidance of the second kind follows an avoidance of the first kind. 
For the purpose of this paper, to generalize the valuation avoidance lemma of Azarang, we need two new concepts very similar to strongly irreducible and pseudo-radical, but in a more restricted sense, as follows.

Definition 2. Let $\mathcal{F}$ be a set of $R$-modules. Then the $R$-module $N$ is said to be strongly irreducible relative $\mathcal{F}$, if the following implication holds

$$
N \supseteq N_{1} \cap \cdots \cap N_{s}, \text { where } N_{i} \in \mathcal{F} \text { for } i=1, \ldots, s \Rightarrow N \supseteq N_{i} \text { for some } i
$$

An $R$-module $L$ which is an intersection of (possibly infinitely many) strongly irreducible modules relative $\mathcal{F}$, is said to be pseudo-radical relative $\mathcal{F}$.

That the two avoidance-situations are related is again established by the proposition which follows.

Proposition 3. Suppose $N \subseteq N_{1} \cup \cdots \cup N_{s}$, where $N_{1}$ is pseudo-radical relative the set $\left\{N, N_{2}, N_{3}, \ldots, N_{s}\right\}$. Then either $N \subseteq N_{1}$ or $N \subseteq N_{2} \cup N_{3} \cup \cdots \cup N_{s}$.

Proof. Let $\mathcal{F}=\left\{N, N_{2}, N_{3}, \ldots, N_{s}\right\}$ and suppose $N \nsubseteq N_{2} \cup N_{3} \cup \cdots \cup N_{s}$. We have, say, $N_{1}=\bigcap L_{i}$, where the $L_{i}$ are strongly irreducible relative $\mathcal{F}$. Fix an $i$ and consider the covering $N \subseteq L_{i} \cup N_{2} \cup N_{3} \cup \cdots \cup N_{s}$, and remove from the covering any superflous $N_{i}$. It is a consequence of Lemma 1 that $N \cap N_{2} \cap N_{3} \cap \cdots \cap N_{s} \subseteq L_{i}$, and hence $N \subseteq L_{i}$ (since $L_{i}$ is strongly irreducible relative $\mathcal{F}$ ). Since this holds for every $L_{i}$, we have $N \subseteq N_{1}$.

Remark. As an immediate application, suppose $N \subseteq N_{1} \cup \cdots \cup N_{s}$, where $N_{i}$ is pseudoradical relative $\left\{N, N_{j} ; j>i\right\}$, for $i=1,2, \ldots, s-2$. Then $N \subseteq N_{i}$ for some $i$. This can be seen as follows. If $N \nsubseteq N_{i}$ for $i \leq s-2$, we can successively delete these $N_{i}$, and obtain $N \subseteq N_{s-1} \cup N_{s}$, and hence $N \subseteq N_{s-1}$ or $N \subseteq N_{s}$.

It is true, that this does not really make sense, unless we have an example of a module being strongly irreducible relative some $\mathcal{F}$, without being strongly irreducible in the more general sense. Before providing such an example (it will appear in Proposition 5), we state without proof a few basic and well-known facts on overrings of an integral domain $R$.

(i) Let $A$ be any overring of $R$. Then $A=\bigcap_{\mathfrak{m}} A_{\mathfrak{m}}$, the intersection taken over all maximal ideals of $A$.

(ii) Let $\mathfrak{p}_{1}, \mathfrak{p}_{2}, \ldots, \mathfrak{p}_{n}$ be prime ideals of $R$, no two being comparable. Let $B=R_{\mathfrak{p}_{1} \cup \ldots \cup \mathfrak{p}_{n}}$ and let $\mathfrak{q}_{i}$ be the extensions of $\mathfrak{p}_{i}, i=1, \ldots, n$ to $B$. Then $\mathfrak{q}_{1}, \mathfrak{q}_{2}, \ldots, \mathfrak{q}_{n}$ are the maximal ideals of $B, R_{\mathfrak{p}_{i}}=B_{\mathfrak{q}_{i}}$ for each $i$, and $B=B_{\mathfrak{q}_{1}} \cap \cdots \cap B_{\mathfrak{q}_{n}}=R_{\mathfrak{p}_{1}} \cap \cdots \cap R_{\mathfrak{p}_{n}}$.

When $N$ is strongly irreducible or pseudo-radical relative a certain $\mathcal{F}$, then this $\mathcal{F}$ can be assumed to be closed under the formation of finite intersections, or in other words we have the following.

Lemma 4. Suppose $N$ is strongly irreducible relative a class $\mathcal{F}$ of $R$-modules. Let $\mathcal{F}^{\prime}$ be the class of finite intersections of modules from $\mathcal{F}$. Then $N$ is strongly irreducible relative $\mathcal{F}^{\prime}$. The same is true with pseudo-radical in place of strongly irreducible. 
Proof. This is rather obvious. Suppose $N$ is strongly irreducible relative $\mathcal{F}$ and suppose $N \supseteq N_{1} \cap \cdots \cap N_{s}$, where each $N_{i} \in \mathcal{F}^{\prime}$, and say $N_{i}=N_{i, 1} \cap \cdots \cap N_{i, k_{i}}$. Then $N \supseteq N_{i, j}$ some $i, j$, and hence $N \supseteq N_{i}$. The result for pseudo-radical $N$ follows as an immediate consequence.

We follow the modern terminology and call a ring with only one maximal ideal a quasi-local ring, and we show next that every quasi-local overring is strongly irreducible relative the class of rings, which are obtained by localizing $R$ at a finite union of prime ideals.

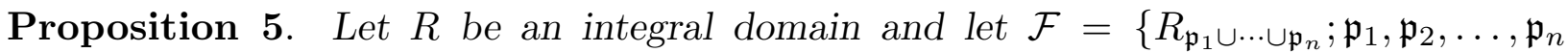
prime ideals of $R\}$. Then every quasi-local overring of $R$ is strongly irreducible relative $\mathcal{F}$, and hence every overring of $R$ is pseudo-radical relative $\mathcal{F}$.

Proof. The second statement follows from the first, since every domain $A$ is the intersection of its localizations $A_{\mathfrak{m}}$. Now, a typical ring in $\mathcal{F}$, is a finite intersection of rings of the form $R_{\mathfrak{p}}$. Thus, by Lemma 4 , it is enough to prove that every quasi-local overring $A$ of $R$ is strongly irreducible relative the smaller class $\left\{R_{\mathfrak{p}} ; \mathfrak{p}\right.$ prime in $\left.R\right\}$. Now suppose that $A$ is quasi-local and that $A \supseteq R_{\mathfrak{p}_{1}} \cap \cdots \cap R_{\mathfrak{p}_{n}}$. Here we may assume that no two $R_{\mathfrak{p}_{i}}$ are comparable. Then no two of the $\mathfrak{p}_{i}$ are comparable and hence $R_{\mathfrak{p}_{1}} \cap \cdots \cap R_{\mathfrak{p}_{n}}=$ $B_{\mathfrak{q}_{1}} \cap \cdots \cap B_{\mathfrak{q}_{n}}$, where $B=R_{\mathfrak{p}_{1}} \cap \cdots \cap R_{\mathfrak{p}_{n}}$, and where $\mathfrak{q}_{1}, \mathfrak{q}_{2}, \ldots, \mathfrak{q}_{n}$ are the maximal ideals of $B$. Let $\mathfrak{m}$ be the maximal ideal of $A$, and put $\mathfrak{p}=B \cap \mathfrak{m}$. Then $B_{\mathfrak{p}} \subseteq A$, but $\mathfrak{p} \subseteq \mathfrak{q}_{i}$, some $i$, and therefore $B_{\mathfrak{p}} \supseteq B_{\mathfrak{q}_{i}}=R_{\mathfrak{p}_{i}}$, and hence $A \supseteq R_{\mathfrak{p}_{i}}$.

We have now all we need to prove a theorem on the avoidance of certain overrings of a domain.

Theorem 6. Suppose $A \subseteq A_{1} \cup \cdots \cup A_{s}$, where $A, A_{1}, A_{2}, \ldots, A_{s}$ are overrings of an integral domain $R$, where $A$ is of the form $R_{\mathfrak{p}_{1} \cup \ldots \cup \mathfrak{p}_{n}}$ for prime ideals $\mathfrak{p}_{i}$ and where each $A_{i}, i \geq 2$ is of the form $S_{i}^{-1} R$ for some multiplicatively closed subset $S_{i}$ of $R$. Then $A \subseteq A_{i}$ for some $i$.

Remark. Note that we suppose that $A$ is $R$ localized at a finite union of prime ideals, whereas the $A_{i}$ may be $R$ localized at a possibly infinite union of prime ideals. On $A_{1}$, there is no restriction at all.

Proof. We begin by proving the theorem in the special case where $A_{i}=R_{\mathfrak{p}_{i}}, i \geq 2$. Then according to Proposition $5, A_{1}$ is pseudo-radical relative $A, A_{2}, A_{3}, \ldots, A_{s}$. Hence, if $A \nsubseteq A_{1}$, we can, by Proposition 3, delete $A_{1}$ from the relation $A \subseteq A_{1} \cup \cdots \cup A_{s}$. Next if also $A \nsubseteq A_{2}$, then $A_{2}$ may be deleted, and proceeding like this we find $A \subseteq A_{i}$ for some $i$. This completes the proof in the special case. In the general case, suppose (in order to derive a contradiction) that $A \nsubseteq A_{i}$ for all $i$. Then there are, for $i=2,3, \ldots, s$, maximal ideals $\mathfrak{m}_{i}$ of $A_{i}$, such that $A \nsubseteq\left(A_{i}\right)_{\mathfrak{m}_{i}}$. Note that $\left(A_{i}\right)_{\mathfrak{m}_{i}}=R_{\mathfrak{n}_{i}}$, where $\mathfrak{n}_{i}=\mathfrak{m}_{i} \cap R$. We have $A \subseteq A_{1} \cup\left(A_{1}\right)_{\mathfrak{m}_{2}} \cup \cdots \cup\left(A_{s}\right)_{\mathfrak{m}_{s}}$, and hence, by the special case just proved, $A \subseteq\left(A_{i}\right)_{\mathfrak{m}_{i}}$ for some $i$, and there is a contradiction.

Remark. The valuation avoidance lemma is a special case of this, since if $V \subseteq V_{1} \cup \cdots \cup V_{n}$, where $V, V_{1}, V_{2}, \ldots, V_{n}$ are all valuation overrings of $R=V_{1} \cap \cdots \cap V_{n}$, then every $V_{i}$ is of the form $R_{\mathfrak{p}_{i}}$. We refer to [4, Theorem 107] for this. 
Note from the proof, that we only used Proposition 5 , with $\mathcal{F}$ being the smaller class of the overrings $R_{\mathfrak{p}}$. Note also that from the theorem proved in the special case where $A_{i}=R_{\mathfrak{p}_{i}}$, $i \geq 2$, we deduced the full theorem. Considering this, a question which comes naturally to ones mind, is whether $\mathcal{F}$ in Proposition 5 could be extended to include every overring of the form $S^{-1} R$. But this is not so, as the following example shows.

Example 7. Let $R=k[x, y]$, where $k$ is a field. Let $A=R_{(y)}$, which is a quasilocal overring of $R$. Next let $S=\left\{x^{n} y^{n} ; n \geq 0\right\}$ and $T=\left\{(x+1)^{n} y^{n} ; n \geq 0\right\}$. Then $S^{-1} R \nsubseteq R_{(y)}, T^{-1} \nsubseteq R_{(y)}$, but $S^{-1} R \cap T^{-1} R=R$, so $S^{-1} R \cap T^{-1} R \subseteq R_{(y)}$. This shows that $R_{(y)}$ is not strongly irreducible relative the class of overrings of the form $S^{-1} R$.

For Bézout domains, Theorem 6 becomes more attractive.

Corollary 8. Let $R$ be a Bézout domain and $A=R_{\mathfrak{p}_{1} \cup \ldots \cup \mathfrak{p}_{n}}$ for prime ideals $\mathfrak{p}_{1}, \mathfrak{p}_{2}, \ldots$, $\mathfrak{p}_{n}$. Further let $A_{1}, A_{2}, \ldots, A_{s}$ be any overrings of $R$. Then if $A \subseteq A_{1} \cup \cdots \cup A_{s}$, in fact $A \subseteq A_{i}$ for some $i$.

Proof. This follows from the fact, which can be readily proved, that every overring of a Bézout domain $R$ is of the form $S^{-1} R$.

\section{References}

1. A. Azarang, The space of maximal subrings of a commutative ring, Comm. Algebra 43 (2015), 795-811.

2. C. Gottlieb, Finite unions of submodules, Comm. Algebra 43 (2015) no. 2, 847-855.

3. W. Heinzer, L. Ratliff, D. Rush, Strongly irreducible ideals of a commutative rings., J. Pure Appl. Algebra 166 (2002), no. 3, 267-275.

4. I. Kaplansky, Commutative rings, The University of Chicago Press, 1970, ISBN 0-22642454-5.

5. N. McCoy, A note on finite unions of ideals and subgroups, Proc. Amer. Math. Soc. 8 (1957), 633-637. 